\title{
Generalized Blockmodeling of Two-mode Network Data
}

\author{
Patrick Doreian \\ University of Pittsburgh, USA
}

\author{
Vladimir Batagelj, Anuška Ferligoj \\ University of Ljubljana, Slovenia
}

September 27, 2003

\begin{abstract}
We extend the direct approach for blockmodeling one-mode data to two-mode data. The key idea in this development is that the rows and columns are partitioned simultaneously but in different ways. Many (but not all) of the generalized block types can be mobilized in blockmodeling twomode network data. These methods were applied to some 'voting' data from the 2000-2001 term of the Supreme Court and to the classic Deep South data on women attending events. The obtained partitions are easy to interpret and compelling. The insight that rows and columns can be partitioned in different ways can be applied also to one-mode data. This is illustrated by a partition of a journal-tojournal citation network where journals are viewed simultaneously as both producers and consumers of scientific knowledge.
\end{abstract}

Blockmodeling tools were developed to partition network actors into clusters, called positions, and, at the same time, to partition the set of ties into blocks that are defined by the positions. (See Lorrain and White (1971), Breiger et al. (1975), and Burt (1976) for the foundational statements.) For these authors, and those using their methods, the foundation for the partitioning was structural equivalence. White and Reitz (1983) generalized structural equivalence to regular equivalence as another principle for blockmodeling networks. For all of these authors, the use of blockmodeling tools was inductive in the sense of specifying an equivalence type and searching for partitions that approximated those equivalence types $^{1}$. The procedures were indirect in the sense of converting network data into a (dis)similarity matrix and using some clustering algorithm. Batagelj et al. (1992a,b) suggested an alternative strategy where the partitioning was done by using the network data directly. In essence, their approach was built upon the recognition that both structural and regular equivalence define certain block types if a partition of actors and ties is exact and consistent with the type of equivalence. For structural equivalence, the ideal blocks are null and complete (Batagelj et al. 1992a), and for regular equivalence, the ideal block types are null and regular (Batagelj et al. 1992b). Subsequently, blockmodeling was generalized to permit many new types of blocks. See Batagelj, 1997 and Doreian et al. (1994). The notion of constructing blockmodeling in terms of a larger set of block types, together with the use of optimization methods mobilized within a direct approach has been called generalized blockmodeling (Doreian et al. (2004). Hitherto, these methods have been applied only to one-mode network data. Here, we consider another extension of blockmodeling by including two-mode network data.

\section{Two-mode Network Data}

Wasserman and Faust (1994: Chapter 8) provide a discussion of affiliation networks as two-mode data. In essence, two-mode data are defined for two sets of social units and contain measurements of a relation from the units in one set to units in the other set. Pairs of network actor types and relations include:

\footnotetext{
${ }^{1}$ An exception is Heil and White (1976) but their algorithm did not enjoy widespread usage for blockmodeling.
} 
people attending events, organizations employing people, justices on a court rendering decisions, and nations belonging to alliances. The most used example of a two-mode network is the Deep South data, also known as the 'Southern Women' data, collected by Davis et al. (1941) for a set of women attending social events over a nine month period. We consider these data shortly as our second example.

\section{Approaches to Two-Mode Network Data}

In a two-mode network, $\mathbf{N}=\left(\mathcal{U}_{1}, \mathcal{U}_{2}, R, w\right)$, one set of social units is denoted by $\mathcal{U}_{1}=\left\{u_{1}, u_{2}, \ldots, u_{n_{1}}\right\}$ and the second set of units is denoted by $\mathcal{U}_{2}=\left\{v_{1}, v_{2}, \ldots, v_{n_{2}}\right\}$. By definition, $\mathcal{U}_{1} \cap \mathcal{U}_{2}=\emptyset$. The social relation $R \subseteq \mathcal{U}_{1} \times \mathcal{U}_{2}$ is defined as one between the units in these two sets and is represented by the set of lines with initial vertices in the set $\mathcal{U}_{1}$ and terminal vertices in the set $\mathcal{U}_{2}$. The mapping $w: R \rightarrow \mathbb{R}$ is a weight. Two examples of weighted two-mode networks are $\left\{\right.$ Persons $\left(\mathcal{U}_{1}\right)$, Goods/Services $\left(\mathcal{U}_{2}\right)$, consumed $(R)$, frequency $(w)\}$ and $\left\{\right.$ Countries $\left(\mathcal{U}_{1}\right)$, Countries $\left(\mathcal{U}_{2}\right)$, imported from $(R)$, value $\left.(w)\right\}$. If no weight is defined we can assume a constant weight $w(u, v)=1$ for all $u R v$.

Many network analytic tools have been used to study the structure contained in two-mode data with distinct ways of representing this type of data. We focus here on the approaches that are primarily algebraic. One takes the form of using a rectangular $n_{1} \times n_{2}$ matrix $\mathbf{A}=\left[a_{u v}\right]$ to represent the data for the two sets of units, $\mathcal{U}_{1}$ and $\mathcal{U}_{2}$.

$$
a_{u v}= \begin{cases}w(u, v) & u R v \\ 0 & \text { otherwise }\end{cases}
$$

This (rectangular) matrix can be binary or valued. For two of the examples that follow, $\mathbf{A}$ has a binary form. The third example that we consider involves valued data.

A two-mode network can be viewed also as an ordinary (one-mode) network on the vertex set $\mathcal{U}_{1} \cup \mathcal{U}_{2}$, divided into two sets $\mathcal{U}_{1}$ and $\mathcal{U}_{2}$, where the arcs can only go from $\mathcal{U}_{1}$ to $\mathcal{U}_{2}$ - it is a bipartite directed graph. Borgatti and Everett (1997: 248) provide a pictorial representation of a bipartite graph for the Southern Women data set and advocate the inclusion of analyses of the bipartite matrix, in this form, in the analysis of two-mode data. Freeman (2003) also displays the Southern Women data in this fashion.

One algebraic approach to two-mode data takes the form of exploring the 'duality' of two-mode data (Breiger, 1974) by constructing two valued one-mode networks. The matrix, $\mathbf{A}_{1}=\mathbf{A} \mathbf{A}^{T}$, where $T$ denotes transposition, is in one-mode form for the actors in $\mathcal{U}_{1}$ and $\mathbf{A}_{2}=\mathbf{A}^{T} \mathbf{A}$ is in one-mode form for the actors in $\mathcal{U}_{2}$. Analyses then are conducted by using these two one-mode representations.

Atkin (1974) introduced Q-Analysis as a way of delineating the structure of the dual simplicial complexes of $\mathbf{A}_{1}$ and $\mathbf{A}_{2}$. Doreian (1979) to analyze the structure of the Southern Women data using these tools. Freeman (1980) provides another example of using Q-Analysis for network data and Seidman (1981) provides a related discussion in terms of hypergraphs. Galois lattices provide another algebraic approach to two-mode data, one taken by Freeman and White (1994) who also applied these methods to the Southern Women data. Once $\mathbf{A}_{1}$ and $\mathbf{A}_{2}$ have been constructed as one-mode matrices, all of the conventional techniques for analyzing one mode data can be used.

\section{Blockmodels for Two-Mode Network Data}

Thinking of applying generalized blockmodeling tools to two-mode data implies making some adjustments to this set of techniques, as well as the thinking behind them. Because the data come in the form of rectangular arrays the language of diagonal and off-diagonal blocks is no longer applicable. Blockmodels applied to (the usual) 'square' network data, require that the rows and columns are partitioned simultaneously in exactly the same way. It makes no sense, conceptually and technically, to partition 
rows and columns of a rectangular array in the same fashion. In this paper, the rows and columns of the rectangular array are partitioned at the same time but they are partitioned differently.

We do not regard the use of partitioning tools for the above matrix representation of the bipartite graph (using $\mathbf{A}, \mathbf{A}^{T}$ and two null matrices) as appropriate. The reason is simple: it is possible to have mixtures of the two distinct types of actors in the same cluster. Indeed, exactly this happened with Borgatti and Everett (1997) partition of the bipartite network in this form for the Southern Women data. We propose the following procedure to ensure that actors of different types are never clustered together: the two-mode data are treated as two-mode data.

We establish a simple notation given $\mathcal{U}_{1}=\left\{u_{1}, u_{2}, \ldots, u_{n_{1}}\right\}$ and $\mathcal{U}_{2}=\left\{v_{1}, v_{2}, \ldots, v_{n_{2}}\right\}$. Let $k_{1}$ be the number of clusters for actors in $\mathcal{U}_{1}$ and let $k_{2}$ the number of clusters of actors in $\mathcal{U}_{2}$. Clearly, $1 \leq k_{1} \leq n_{1}$ and $1 \leq k_{2} \leq n_{2}$. While it is possible to consider up to $n_{1}$ and $n_{2}$ for the number of clusters of the two sets of actors, the point of the blockmodeling effort is to use fewer clusters than actors. We will label a partition with $k_{1}$ clusters of the actors in $\left(\mathcal{U}_{1}\right)$ and $k_{2}$ clusters of the actors in $\left(\mathcal{U}_{2}\right)$ as a $\left(k_{1}, k_{2}\right)$-partition of the two-mode array.

\section{A Formalization of Blockmodeling Two-mode Data}

The theoretical background for two-mode blockmodeling comes from Bategelj et al. (1992a,b), Doreian et al. (1994) and Batagelj et al. (1998): we view the blockmodeling of two-mode data as a simple extension of one-mode blockmodeling.

The main difference is that in blockmodeling of a two-mode network $\mathbf{N}=\left(\mathcal{U}_{1}, \mathcal{U}_{2}, R, w\right)$ we are trying to identify a two-clustering $\mathbf{C}=\left(\mathbf{C}_{1}, \mathbf{C}_{2}\right)-\mathbf{C}_{1}$ is a partition of $\mathcal{U}_{1}$ and $\mathbf{C}_{2}$ is a partition of $\mathcal{U}_{2}-$ such that they induce selected blocks. We denote the set of all feasible two-clusterings with $\Phi$.

The two-mode generalized problem can be formulated as an optimization problem $(\Phi, P, \min )$ :

Determine the two-clustering $\mathbf{C}^{\star}=\left(\mathbf{C}_{1}^{\star}, \mathbf{C}_{2}^{\star}\right) \in \Phi$ for which

$$
P\left(\mathbf{C}^{\star}\right)=\min _{\mathbf{C} \in \Phi} P(\mathbf{C})
$$

where $\Phi$ is the set of feasible two-clusterings and $P$ is the criterion function.

The criterion function $P(\mathbf{C})$ is obtained in the same way as in the ordinary case:

$$
P(\mathbf{C})=P\left(\left(\mathbf{C}_{1}, \mathbf{C}_{2}\right)\right)=\sum_{C_{u} \in \mathbf{C}_{1}, C_{v} \in \mathbf{C}_{2}} \min _{B \in \mathcal{B}\left(C_{u}, C_{v}\right)} \delta\left(R\left(C_{u}, C_{v}\right), B\right)
$$

where $\mathcal{B}\left(C_{u}, C_{v}\right), C_{u} \subseteq \mathcal{U}_{1}$ and $C_{v} \subseteq \mathcal{U}_{2}$ denotes the set of all ideal blocks corresponding to block $R\left(C_{u}, C_{v}\right)$, and the term $\delta\left(R\left(C_{u}, C_{v}\right), B\right)$ measures the difference (number of inconsistencies) between the block $R\left(C_{u}, C_{v}\right)$ and the ideal block $B$. The value of $\delta$ is constructed on the basis of the characterizations of the types of blocks in such as fashion that makes $\delta$ compatible with the selected type of equivalence. (See Batagelj et al. (1992a).) The criterion function $P\left(\left(\mathbf{C}_{1}, \mathbf{C}_{2}\right)\right)$ is sensitive iff $P\left(\left(\mathbf{C}_{1}, \mathbf{C}_{2}\right)\right)=0 \Leftrightarrow$ the blockmodel determined by $\left(\mathbf{C}_{1}, \mathbf{C}_{2}\right)$ is exact.

The resulting optimization problem can be solved by a local optimization procedure as follows:

Determine the initial clustering $\mathbf{C}$;

repeat:

if in the neighborhood of the current clustering $\mathbf{C}$

there exists a clustering $\mathbf{C}^{\prime}$ such that $P\left(\mathbf{C}^{\prime}\right)<P(\mathbf{C})$

then move to clustering $\mathbf{C}^{\prime}$. 
Usually, the neighborhood is determined by two transformations: moving a unit from one cluster to another cluster; interchanging of two units between different clusters. The procedure is repeated for many initial clusterings and the (those) partition(s) with the smallest value of the criterion function are selected. Once the partitions $\left(\mathbf{C}_{1}, \mathbf{C}_{2}\right)$ and types of blocks are determined, we can also compute the values of connections by using averaging rules.

\section{Three Empirical Examples}

We consider three examples. One features some of the some decisions handed down by the U.S. Supreme Court in their 2000-2001 term. The second comes in the form of the Deep South data discussed above and the third is a journal-to-journal citation network.

\subsection{Supreme Court Voting}

These data come from the simple (preliminary) study by Doreian and Fujimoto (2003) of the Supreme Court Justices and their 'votes' on a set of 26 "important decisions". These data are presented in Table 1. The nine Justices (in the order in which they joined the Supreme Court) are Rehnquist (1972), Stevens (1975), O'Conner (1981), Scalia (1982), Kennedy (1988), Souter (1990), Thomas (1991), Ginsburg (1993) and Breyer (1994). The distribution of votes are organized in terms of the substantive content of the selection of decisions as organized by Greenhouse (2001).

Each row of Table 1 represents a decision handed down by the Supreme Court. On the left is a descriptive label for that decision. A much fuller description of these case can be found in Greenhouse (2001) and in Doreian and Fujimoto (2003). The substantive details of these cases, while stark, are of secondary concern here. The columns correspond to the nine Justices where the Justices are represented by a label: Breyer (Br), Ginsburg (Gi), Souter (So), Stevens (St), O'Connor (OC), Kennedy (Ke), Rehnquist (Re), Scalia ( $\mathrm{Sc}$ ) and Thomas (Th). In these data, a ' + ' in the column of a Justice represents voting in the majority for that issue and a '-' represents voting in the minority for that decision. The decisions range from the unanimous decision ${ }^{2}$ in the case involving the Clean Air Act to many 5-4 decisions. The latter suggest an ideologically divided court.

In the usual representation of such data, $\mathbf{A}$ is written with the actors (in this case the justices) in the rows and the events (in this case the decisions) in the columns. Table 1 has been written as the transposed form for formatting reasons. In terms of the analysis that follows, rows of $\mathbf{A}$ represent the justices and the columns their decisions.

Doreian and Fujimoto viewed these data as 'signed' two-mode data. They constructed a matrix, $\mathbf{A}_{p}$, for majority voting where 1 represented a majority vote and 0 a vote that was not for the majority (i.e. either a vote against the decision or an abstention). Similarly, they constructed a matrix $\mathbf{A}_{n}$ for the negative votes for a decision, where 1 represented a vote in the minority and 0 represented either an abstention or a vote with the majority. Their analyses involved (for a focus on the justices) $\mathbf{A}_{p} \mathbf{A}_{p}^{T}$ for majority voting, $\mathbf{A}_{n} \mathbf{A}_{n}^{T}$ for minority voting and $\mathbf{A}_{t} \mathbf{A}_{t}^{T}$ where $\mathbf{A}_{t}=\mathbf{A}_{p}+\mathbf{A}_{n}$ for all voting. Their analyses were designed to explore the differing patterns of joint majority voting, joint minority voting and joint overall voting patterns. They used a combination of tools including Q-Analysis and partitions based on structural equivalence for the generated one-mode matrices. Some nuanced interpretations follow as a result of looking at the differences between majority and minority voting patterns. As a result of these different analyses, they reached a partition - having four clusters of justices and eight clusters of

\footnotetext{
${ }^{2}$ There is one case that was unanimous except for Breyer who abstained because his brother had ruled on the case at a lower court level.
} 
Table 1: Supreme Court Voting for Twenty-Six Important Decisions

\begin{tabular}{|c|c|c|c|c|c|c|c|c|}
\hline Issue & $\mathrm{Br} \mathrm{Gi}$ & So & $\mathrm{St}$ & $\mathrm{OC}$ & $\mathrm{Ke}$ & $\operatorname{Re}$ & $\mathrm{Sc}$ & Th \\
\hline Presidential Election & - & - & - & + & + & + & + & + \\
\hline \multicolumn{9}{|c|}{ Criminal Law Cases } \\
\hline Illegal Search 1 & $+\quad+$ & + & & + & + & - & - & - \\
\hline Illegal Search 2 & $+\quad+$ & + & + & + & + & - & - & - \\
\hline Illegal Search 3 & $+\quad+$ & + & - & - & - & - & + & + \\
\hline Seat Belts & $-\quad-$ & + & - & - & + & + & + & + \\
\hline Stay of Execution & $+\quad+$ & + & + & + & + & - & - & - \\
\hline \multicolumn{9}{|c|}{ Federal Authority Cases } \\
\hline Federalism & $-\quad-$ & - & - & + & + & + & + & + \\
\hline Clean Air Act & $+\quad+$ & + & + & + & + & + & + & + \\
\hline Clean Water & - & - & - & + & + & + & + & + \\
\hline Cannabis for Health & $0+$ & + & + & + & + & + & + & + \\
\hline United Foods & - & + & + & - & + & + & + & + \\
\hline NY Times Copyrights & $-\quad+$ & + & - & + & + & + & + & + \\
\hline \multicolumn{9}{|c|}{ Civil Rights Cases } \\
\hline Voting Rights & $+\quad+$ & + & + & + & - & - & - & - \\
\hline Title VI Disabilities & - & - & - & + & + & + & + & + \\
\hline PGA v. Handicapped Player & $+\quad+$ & + & + & + & + & + & - & - \\
\hline \multicolumn{9}{|c|}{ Immigration Law Cases } \\
\hline Immigration Jurisdiction & $+\quad+$ & + & & - & + & - & - & - \\
\hline Deporting Criminal Aliens & $+\quad+$ & + & + & + & - & - & - & - \\
\hline Detaining Criminal Aliens & $+\quad+$ & + & + & - & + & - & - & - \\
\hline Citizenship & $-\quad-$ & - & + & - & + & + & + & + \\
\hline \multicolumn{9}{|c|}{ Speech and Press Cases } \\
\hline Legal Aid for Poor & $+\quad+$ & + & + & - & + & - & - & - \\
\hline Privacy & $+\quad+$ & + & + & + & + & - & - & - \\
\hline Free Speech & $+\quad-$ & - & - & + & + & + & + & + \\
\hline Campaign Finance & $+\quad+$ & + & + & + & - & - & - & - \\
\hline Tobacco Ads & $-\quad-$ & - & - & + & + & + & + & + \\
\hline \multicolumn{9}{|c|}{ Labor and Property Rights Cases } \\
\hline Labor Rights & $-\quad-$ & - & - & + & + & + & + & + \\
\hline Property Rights & - & - & - & + & + & + & + & + \\
\hline
\end{tabular}

decisions - with 13 inconsistencies with an ideal partition based on structural equivalence ${ }^{3}$. Here, we focus on the two-mode data as given in Table 1 and use a direct partition of the two-mode data with four clusters of the justices and seven clusters of the decisions ${ }^{4}$ : i.e. a $(4,7)$-partition in the notation of Section 3. The clustering of the justices is identical with the partition reported by Doreian and Fujimoto. The difference between to two procedures comes with the partition of the decisions.

Figure 1 shows the fitted blockmodel for structural equivalence where four (4) clusters were specified for the justices and seven (7) clusters were specified for the for the decisions.

For the justices, two of the four clusters are \{Breyer, Ginsburg, Souter, Stevens\}, and \{Rehnquist,

${ }^{3}$ The value of the criterion function for their reported partition was computed for the partition they reported and was not 


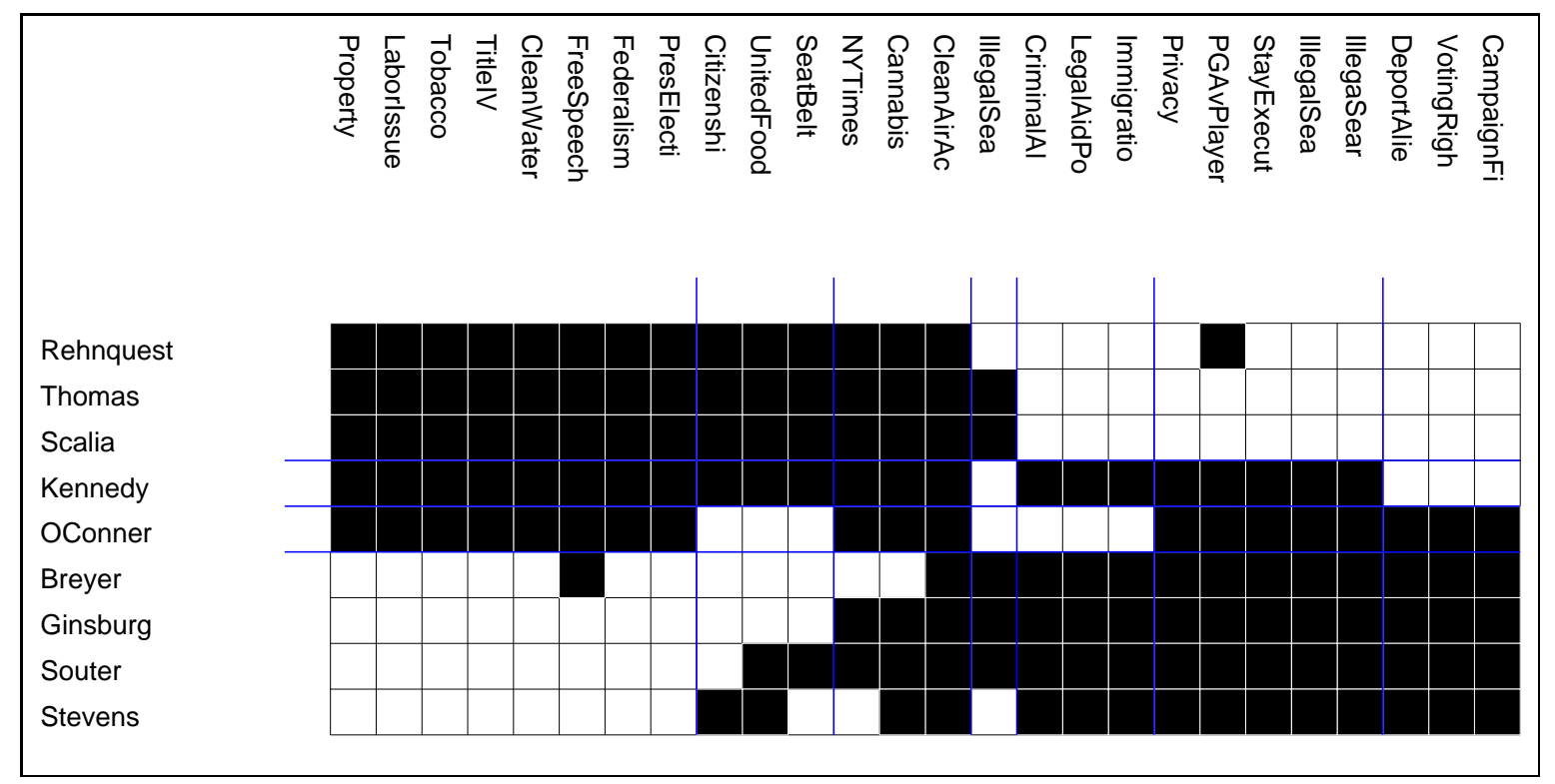

Figure 1: A (4,7)-partition of the Supreme Court Voting

Scalia, Thomas $\}$ that can be viewed, respectively, as the liberal and conservative wings of the Supreme Court (Doreian and Fujimoto, 2003). The two singletons, O'Connor and Kennedy can be interpreted in several ways. In one interpretation, they can be seen as justices that form a bridge between the two wings. Alternatively, they can be viewed as being a part of the conservative wing that joins with the liberal wing on some of the issues that come before the court. Kennedy is the justice who most often is a part of the majority (22 times in the 26 decisions considered here). O'Connor is in the majority for 19 of the decisions ${ }^{5}$.

For the set of 26 decisions, the first cluster on the left (on top) in Figure 1 are decisions where the conservative core plus O'Connor and Kennedy won with a narrow 5-4 vote plus the Free Speech case where Breyer joins the majority. His vote on this issue is an inconsistency with a perfect structural equivalence partition. The second cluster of cases (on the left) has the Seat Belt, United Foods and the Citizenship cases. For these decisions, the conservative core plus Kennedy are joined by justices from the liberal wing. Justices Souter and Stevens each joins them twice. These four votes from the liberal wing are all inconsistent with the ideal null block specified under structural equivalence for these three cases. They are the black squares in the block defined by these decisions and the liberal wing cluster. Justice O'Connor's votes are all consistent with a structural equivalence partition for these three cases because she was a singleton and voted in the minority for each of these cases. The third cluster from the left has the cases concerning the Clean Air Act, Cannabis and the New York Times cases. The conservative core of Rehnquist, Thomas and Scalia are joined by both Kennedy and O'Connor together with at least one member of the liberal wing of the court. The inconsistences with structural equivalence are the white squares in the block defined by these cases and the liberal wing.

The remaining clusters of cases are those where the three justices of the conservative core are not a part of the majority (except for Rehnquist with the PGA versus a handicapped player). The singleton, Illegal Search 3, is a most improbable decision where Scalia and Thomas are joined by three members of

\footnotetext{
used as a part of their partitioning methods.

${ }^{4}$ In selecting this particular partition we were helped by the partition reported by Doreian and Fujimoto (2003).

${ }^{5}$ These two justices are differentiated by Doreian and Fujimoto (2003) in terms of minority voting, $A_{n}$, where O'Connor has a bridging role not shared by Kennedy.
} 


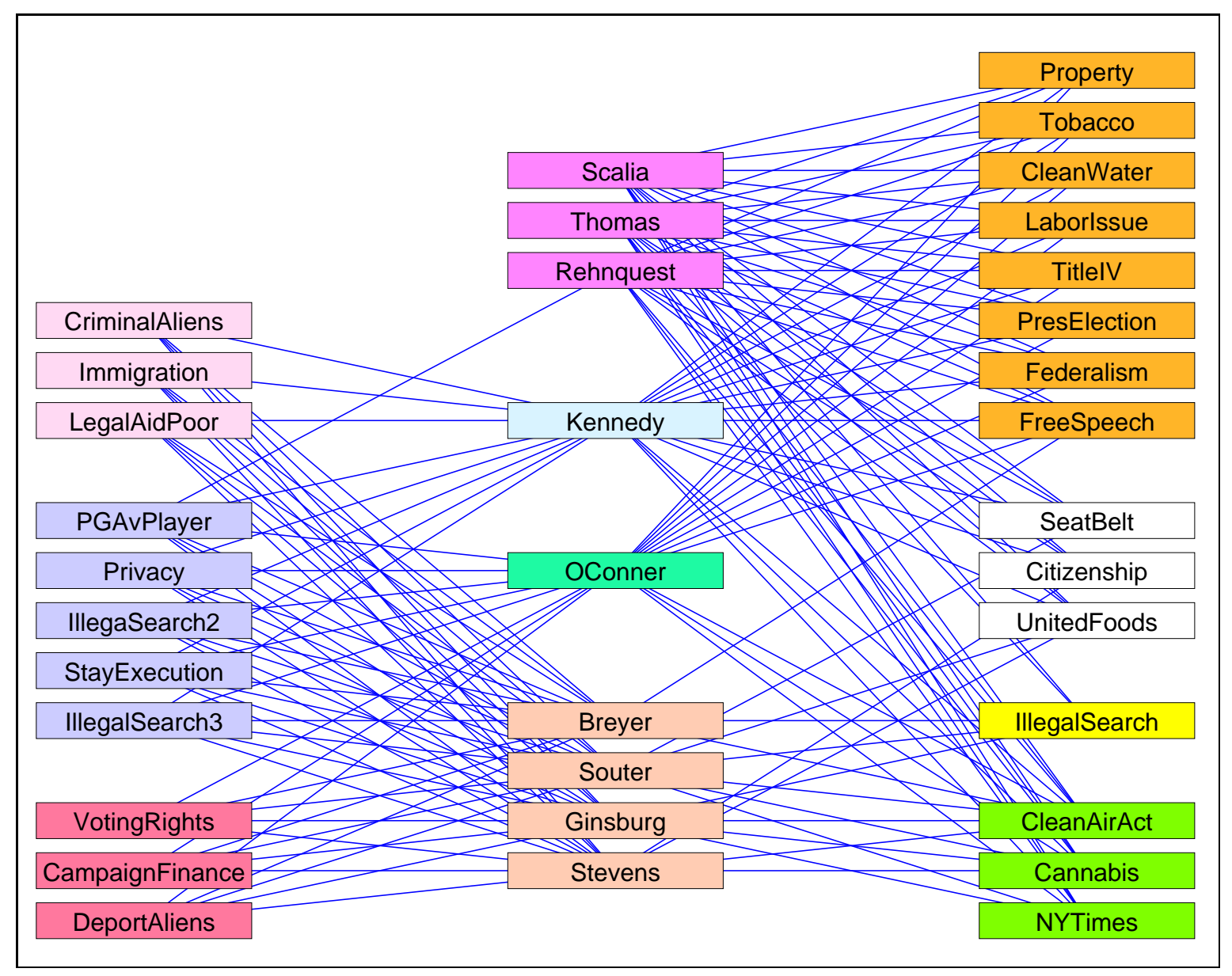

Figure 2: The Bipartite Supreme Court Network with the(4,7)-partition

the liberal wing in a yet another 5-4 decision. The white squares for Rehnquist and Stevens are the inconsistencies with structural equivalence. Next comes three decisions - Legal Aid for the Poor, Detaining Criminal Aliens and Immigration Jurisdiction - where Kennedy joins the liberal wing to produce more 5-4 decisions. There are no inconsistencies with structural equivalence. Next comes five decisions where the liberal wing is joined by both Kennedy and O'Connor ${ }^{6}$. Rehnquist's vote is inconsistent with structural equivalence. Finally, there are three decisions where the liberal wing is joined by O'Connor. These are the Campaign Finance, Voting Rights, and the Departing Aliens cases. There are no inconsistencies with structural equivalence.

Figure 2 provides a pictorial representation of the partitioned two-mode Supreme Court data. The clusters that are produced are homogenous - there is no mixing of the two types of social actors in them. Finally, together, the matrix display in Figure 1 and the picture of the bipartite graph in Figure 2 provide clean displays of the partitioned two-mode data. Of course, Figure 2 shows the bipartite graph.

Having a partition based on structural equivalence, without really pre-specifying a model, that is sufficient to partition the Supreme Court two-mode data set is appealing. However, in general, using just these two types of blocks (null and complete) that could appears anywhere in a blockmodel will not be sufficient to blockmodel all two-mode data structures. We turn now to consider other types of blockmodels and strategies for modeling two-mode data.

\footnotetext{
${ }^{6}$ As noted above, the case of the PGA versus a handicapped player has Rehnquist in the majority.
} 
Table 2: Sorted Participation Matrix

\begin{tabular}{|l|ccccc|cccc|ccccc|}
\hline Actor & $E_{1}$ & $E_{2}$ & $E_{3}$ & $E_{4}$ & $E_{5}$ & $E_{6}$ & $E_{7}$ & $E_{8}$ & $E_{9}$ & $E_{10}$ & $E_{11}$ & $E_{12}$ & $E_{13}$ & $E_{14}$ \\
\hline Evelyn & 1 & 1 & 1 & 1 & 1 & 1 & 0 & 1 & 1 & 0 & 0 & 0 & 0 & 0 \\
Laura & 1 & 1 & 1 & 0 & 1 & 1 & 1 & 1 & 0 & 0 & 0 & 0 & 0 & 0 \\
Theresa & 0 & 1 & 1 & 1 & 1 & 1 & 1 & 1 & 1 & 0 & 0 & 0 & 0 & 0 \\
Brenda & 1 & 0 & 1 & 1 & 1 & 1 & 1 & 1 & 0 & 0 & 0 & 0 & 0 & 0 \\
Charlotte & 0 & 0 & 1 & 1 & 1 & 0 & 1 & 0 & 0 & 0 & 0 & 0 & 0 & 0 \\
Frances & 0 & 0 & 1 & 0 & 1 & 1 & 0 & 1 & 0 & 0 & 0 & 0 & 0 & 0 \\
Eleanor & 0 & 0 & 0 & 0 & 1 & 1 & 1 & 1 & 0 & 0 & 0 & 0 & 0 & 0 \\
Pearl & 0 & 0 & 0 & 0 & 0 & 1 & 0 & 1 & 1 & 0 & 0 & 0 & 0 & 0 \\
Ruth & 0 & 0 & 0 & 0 & 1 & 0 & 1 & 1 & 1 & 0 & 0 & 0 & 0 & 0 \\
\hline Verne & 0 & 0 & 0 & 0 & 0 & 0 & 1 & 1 & 1 & 0 & 0 & 1 & 0 & 0 \\
Myra & 0 & 0 & 0 & 0 & 0 & 0 & 0 & 1 & 1 & 1 & 0 & 1 & 0 & 0 \\
Katherine & 0 & 0 & 0 & 0 & 0 & 0 & 0 & 1 & 1 & 1 & 0 & 1 & 1 & 1 \\
Sylvia & 0 & 0 & 0 & 0 & 0 & 0 & 1 & 1 & 1 & 1 & 0 & 1 & 1 & 1 \\
Nora & 0 & 0 & 0 & 0 & 0 & 1 & 1 & 0 & 1 & 1 & 1 & 1 & 1 & 1 \\
Helen & 0 & 0 & 0 & 0 & 0 & 0 & 1 & 1 & 0 & 1 & 1 & 1 & 0 & 0 \\
Dorothy & 0 & 0 & 0 & 0 & 0 & 0 & 0 & 1 & 1 & 0 & 0 & 0 & 0 & 0 \\
Olivia & 0 & 0 & 0 & 0 & 0 & 0 & 0 & 0 & 1 & 0 & 1 & 0 & 0 & 0 \\
Flora & 0 & 0 & 0 & 0 & 0 & 0 & 0 & 0 & 1 & 0 & 1 & 0 & 0 & 0 \\
\hline
\end{tabular}

\subsection{The Southern Women Event Participation Data}

Our second example is the Davis et al. (1941) Southern Women participating in social events data set. They are shown in Table 2 where a 1 indicates attendance at an event and 0 indicates not attending an event.

As Freeman (2002) notes, this data set ".. reappears whenever any network analyst wants to explore the utility of some new tool for analyzing data." We join the long line of analysts who have mined these data. Our assessment of the utility of using blockmodeling tools for two-mode data has been made much easier by Freeman's (2002) meta-analysis of 21 analyses of these data. His analysis provides a consensual (induced) criterion regarding the 'true structure' in these data. It is also a point of departure for other partitioning strategies.

Freeman is clear that the 'true structure' of the Southern Women data on the participation in social events is one where there are two subgroups. One is composed of \{Evelyn, Laura, Theresa, Brenda, Charlotte, Frances, Eleanor, Pearl, Ruth\} and the other has \{Verne, Myra, Katherine, Sylvia, Nora, Helen, Dorothy, Olivia, Flora $\}$ as its members. These two subgroups of women are labeled as 'Group A' and 'Group B'. Freeman is silent about the corresponding partition of the set of events. We label the events $E_{j}$ for $1 \leq j \leq 14$ where the labeling of the events is consistent with the partitions of those events that we present. We have divided them into three clusters that almost have the form: events $E_{1}$ through $E_{5}$ were events attended only by women of Group A; events $E_{6}$ through $E_{9}$ were events attended by both groups of women and events $E_{10}$ through $E_{14}$ were attended only by the women in Group B. Pearl is the exception in Group A because she attends only $E_{6}, E_{8}$ and $E_{9}$ and Dorothy, by attending only $E_{8}$ and $E_{9}$, is the exception in Group B. This partition is labeled 'Version 1' and is shown as Table 2. Consistent with that description, Figure 3 shows a picture of the bipartite graph with these two clusters of women and the three clusters of events. 


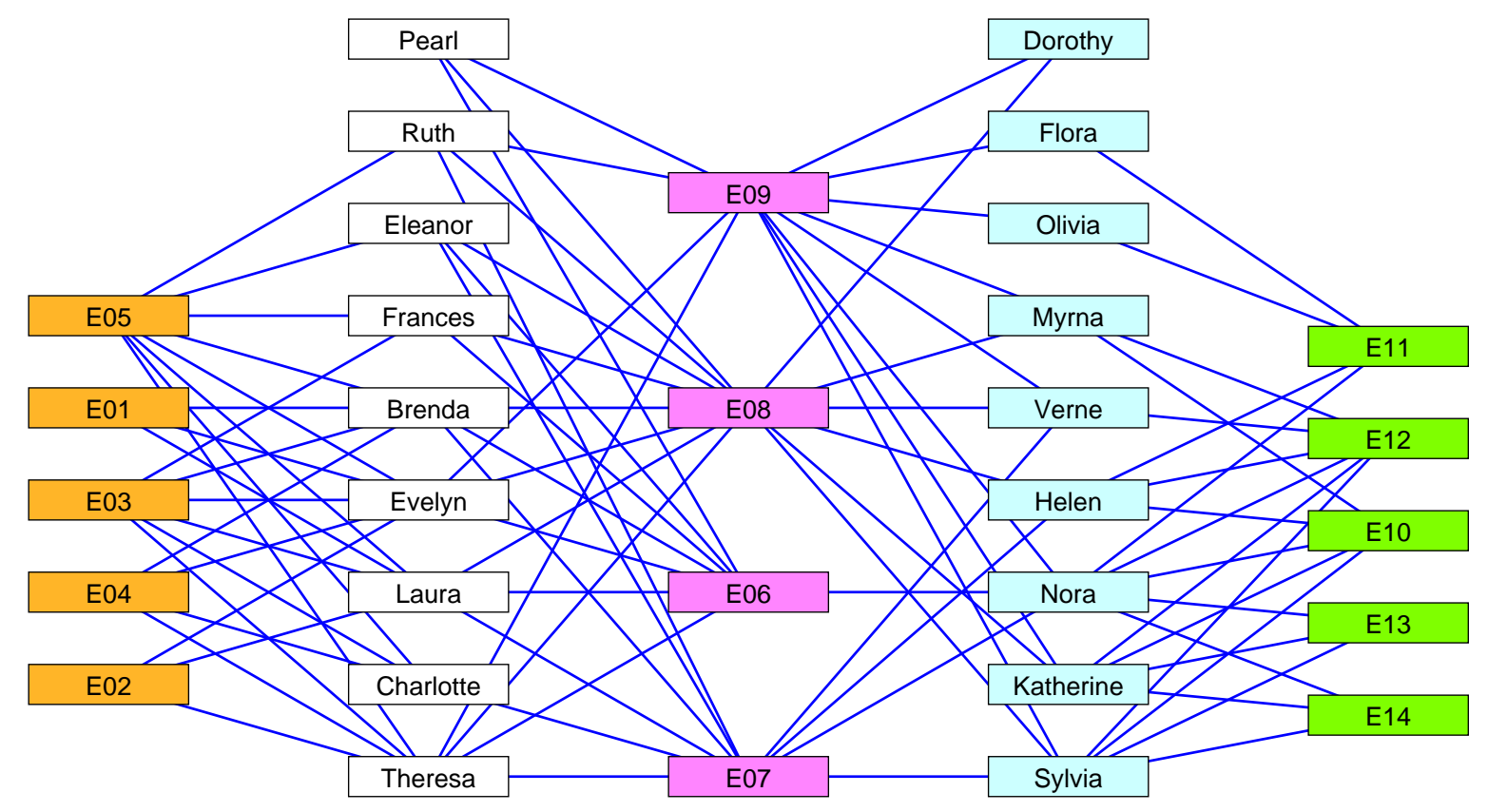

Figure 3: Southern Women Bipartite Network: Version 1

Can the (2,3)-partition shown in Table 2 be recovered as a unique (or near unique) solution by means of (generalized) blockmodeling tools for two-mode data? If only the block types are specified and there are no further specifications, then the answer is no. It becomes necessary to pre-specify (Batagelj et al. (1998) the location of specific block types and it is possible to impose different penalties on some inconsistencies relative to others. Moving in this direction is aided by the summary of the event participation as shown in Table 3.

Table 3 makes it clear that there are events attended only by women from Group A and a set of events attended only by women from Group B. the blockmodel suggested by the counts in Table 3 is:

\begin{tabular}{|ccc|}
\hline com & com & nul \\
nul & com & com \\
\hline
\end{tabular}

With structural equivalence in mind, the specification can be 'strengthened' in the sense of pre-specifying a model in terms of block types and their location within the image. In this case, if there are events attended only be women of Group A and events attended only by women of Group B then there are clear null blocks. It seems sensible to use this information about the structure of the data in Table 2 . So, in addition to specifying that only null and complete blocks are allowed, we can go further and specify where in a blockmodel these null blocks should be located and specify that inconsistencies in the form of $1 \mathrm{~s}$ in these null blocks are to penalized. That is, $1 \mathrm{~s}$ in these null blocks are more consequential than $0 \mathrm{~s}$ in the otherwise complete blocks. The penalty we imposed for 1s in null blocks was 100 with the expectation that null blocks would be identified as null but with the consequence of increasing the number of 0 s in blocks whose corresponding ideal block is complete.

Fitting this block model type, with the penalty, does yield a unique blockmodel with the specified form and 64 inconsistencies with the corresponding ideal blockmodel. The partition for the women is the 'best' partition emerging from Freeman's meta-analysis. The partition of the events is problematic where the partition is $\left\{E_{1}, E_{2}, E_{4}\right\},\left\{E_{3}, E_{5}-E_{9}\right\}$ and $\left\{E_{10}-E_{14}\right\}$. We note that all of the structural equivalence partitions - where only block types were specified - has optimized criterion functions of 53 . 
Table 3: Counts of Participation in Events

\begin{tabular}{|l|c|c|c|}
\hline Actor & $E_{1}-E_{5}$ & $E_{6}-E_{9}$ & $E_{10}-E_{14}$ \\
\hline Evelyn & 5 & 3 & 0 \\
Laura & 4 & 3 & 0 \\
Theresa & 4 & 4 & 0 \\
Brenda & 4 & 3 & 0 \\
Charlotte & 3 & 1 & 0 \\
Frances & 2 & 2 & 0 \\
Eleanor & 1 & 3 & 0 \\
Pearl & 0 & 3 & 0 \\
Ruth & 1 & 4 & 0 \\
\hline Verne & 0 & 3 & 1 \\
Myra & 0 & 2 & 2 \\
Katherine & 0 & 2 & 4 \\
Sylvia & 0 & 3 & 4 \\
Nora & 0 & 3 & 5 \\
Helen & 0 & 2 & 3 \\
Dorothy & 0 & 2 & 0 \\
Olivia & 0 & 1 & 1 \\
Flora & 0 & 1 & 1 \\
\hline
\end{tabular}

By making sure that the null blocks were null, the 'total' number of inconsistencies was raised in the form of 0 s in complete blocks. This is not surprising because seeking partitions with additional constraints (in the pre-specification), in general, leads to partitions with larger values for the criterion function. There is a price to pay if constraints are placed on solutions. In this case, there are null blocks that must be preserved and preserving them for all fitted blockmodels increases the inconsistency count from 53 to 64 . It is a price we are willing to pay and insist that it is a price we often must pay to delineate meaningful partitions if we know more about the data than the presence of blocks consistent with an equivalence type.

The partition shown in Figure 3 and Table 2 is appealing and reproduces the 'truth' identified in Freeman's (2003) meta-analysis of previous analyses of the Davis data. Yet there is a problem in the patten of ties for Pearl and Dorothy. They attend only events common to Group A and Group B. This suggests the partition shown in Table 4 where Pearl and Dorothy form their own group. This suggests a blockmodel with a pre-specified structure of:

\begin{tabular}{|ccc|}
\hline com & com & nul \\
nul & com & com \\
nul & com & nul \\
\hline
\end{tabular}

When this pre-specified model is fitted, a unique solution is returned for the blockmodeling problem for these data and it is exactly the partition shown in Table 4. This blockmodel is shown in Figure 4

The partition shown in Table 4 can be reached in at least two other ways. Doreian et al. (2004) show that both of the following specifications also produce exactly the same partitions of women and events:

\begin{tabular}{|lll|}
\hline reg & reg & nul \\
nul & reg & reg \\
nul & rre & nul
\end{tabular}$\quad$\begin{tabular}{llll|} 
rdo & rdo & nul \\
nul & rdo & rdo \\
nul & cdo & nul \\
\hline
\end{tabular}


Table 4: Sorted Participation Matrix

\begin{tabular}{|l|ccccc|cccc|ccccc|}
\hline Actor & $E_{1}$ & $E_{2}$ & $E_{3}$ & $E_{4}$ & $E_{5}$ & $E_{6}$ & $E_{7}$ & $E_{8}$ & $E_{9}$ & $E_{10}$ & $E_{11}$ & $E_{12}$ & $E_{13}$ & $E_{14}$ \\
\hline Evelyn & 1 & 1 & 1 & 1 & 1 & 1 & 0 & 1 & 1 & 0 & 0 & 0 & 0 & 0 \\
Laura & 1 & 1 & 1 & 0 & 1 & 1 & 1 & 1 & 0 & 0 & 0 & 0 & 0 & 0 \\
Theresa & 0 & 1 & 1 & 1 & 1 & 1 & 1 & 1 & 1 & 0 & 0 & 0 & 0 & 0 \\
Brenda & 1 & 0 & 1 & 1 & 1 & 1 & 1 & 1 & 0 & 0 & 0 & 0 & 0 & 0 \\
Charlotte & 0 & 0 & 1 & 1 & 1 & 0 & 1 & 0 & 0 & 0 & 0 & 0 & 0 & 0 \\
Frances & 0 & 0 & 1 & 0 & 1 & 1 & 0 & 1 & 0 & 0 & 0 & 0 & 0 & 0 \\
Eleanor & 0 & 0 & 0 & 0 & 1 & 1 & 1 & 1 & 0 & 0 & 0 & 0 & 0 & 0 \\
Ruth & 0 & 0 & 0 & 0 & 1 & 0 & 1 & 1 & 1 & 0 & 0 & 0 & 0 & 0 \\
\hline Verne & 0 & 0 & 0 & 0 & 0 & 0 & 1 & 1 & 1 & 0 & 0 & 1 & 0 & 0 \\
Myra & 0 & 0 & 0 & 0 & 0 & 0 & 0 & 1 & 1 & 1 & 0 & 1 & 0 & 0 \\
Katherine & 0 & 0 & 0 & 0 & 0 & 0 & 0 & 1 & 1 & 1 & 0 & 1 & 1 & 1 \\
Sylvia & 0 & 0 & 0 & 0 & 0 & 0 & 1 & 1 & 1 & 1 & 0 & 1 & 1 & 1 \\
Nora & 0 & 0 & 0 & 0 & 0 & 1 & 1 & 0 & 1 & 1 & 1 & 1 & 1 & 1 \\
Helen & 0 & 0 & 0 & 0 & 0 & 0 & 1 & 1 & 0 & 1 & 1 & 1 & 0 & 0 \\
Olivia & 0 & 0 & 0 & 0 & 0 & 0 & 0 & 0 & 1 & 0 & 1 & 0 & 0 & 0 \\
Flora & 0 & 0 & 0 & 0 & 0 & 0 & 0 & 0 & 1 & 0 & 1 & 0 & 0 & 0 \\
\hline Pearl & 0 & 0 & 0 & 0 & 0 & 1 & 0 & 1 & 1 & 0 & 0 & 0 & 0 & 0 \\
Dorothy & 0 & 0 & 0 & 0 & 0 & 0 & 0 & 1 & 1 & 0 & 0 & 0 & 0 & 0 \\
\hline
\end{tabular}

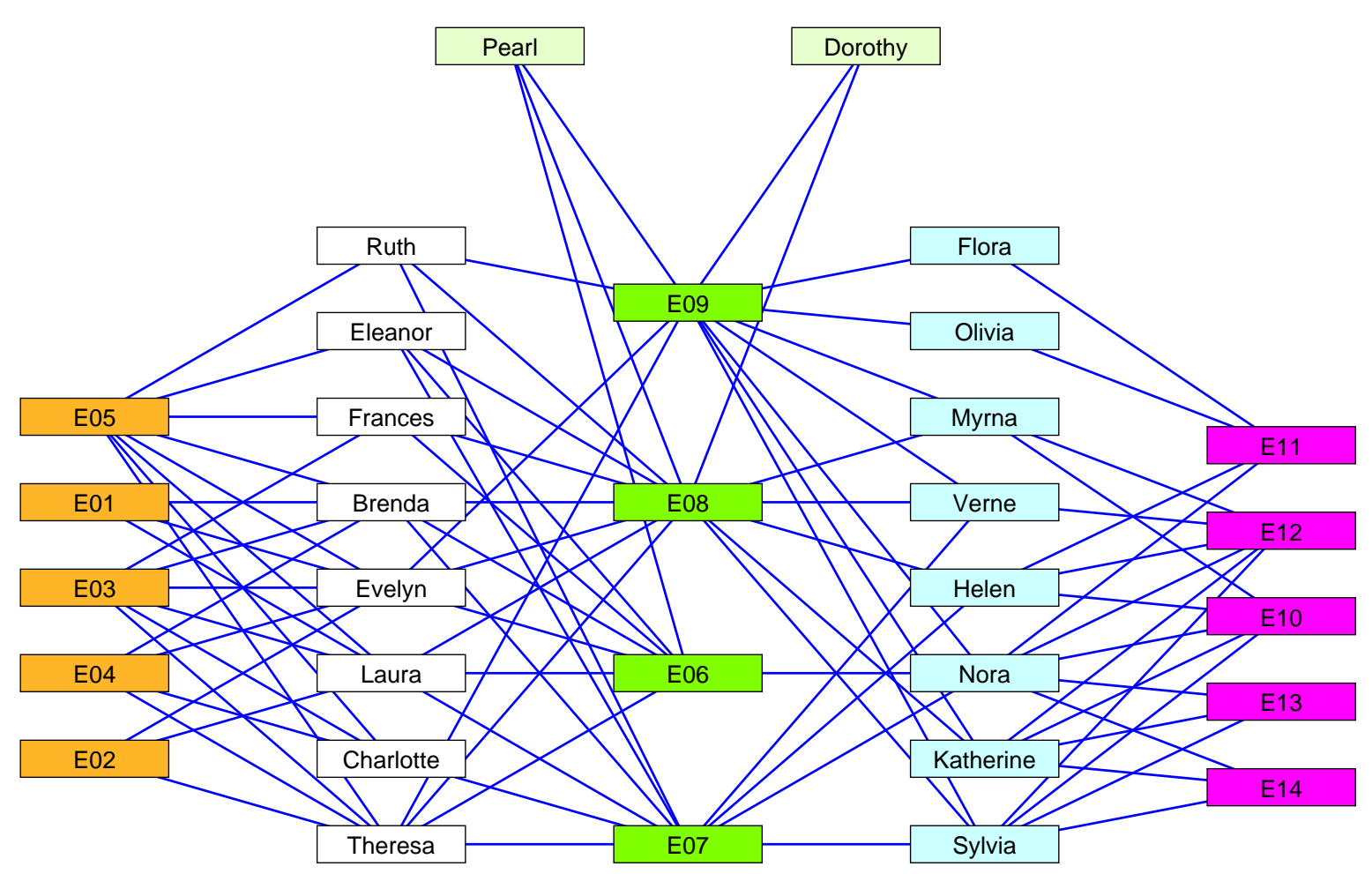

Figure 4: Southern Women Bipartite Network: Version 2 
An examination of Table 4 makes it clear that these are both appropriate pre-specified blockmodels for these data. For both, the same heavy penalty for $1 \mathrm{~s}$ in null blocks was imposed. Of course, the measures of inconsistency change with the change in the definition of block types. See Doreian et al. (2004) for a discussion of having multiple blockmodel types for complementary interpretations of a body of data and a discussion of why inconsistencies for different block types and blockmodels cannot be compared directly.

We turn now to consider delineating the subgroup structure of Groups A and B. Given the robustness of the partition of events into three clusters, we first consider $(5,3)$-partitions. We know already that Dorothy and Pearl belong to neither Group A nor Group B. As a result, we need to specify a cluster for them. If Group $\mathrm{A}$ and $\mathrm{B}$ are each to be split into two subgroups, then $k_{1}$ is 5 . All our attempts to get subgroups of $\mathrm{A}$ and $\mathrm{B}$ with predicates of complete, regular, row-regular and null blocks were not successful. Either there are multiple equally well fitting partitions or the unique partitions, when they are returned, are less than satisfying. The unique partitions most often break Group A into a singleton (usually Evelyn) and the rest of A and to break Group B into a singleton (usually Nora) and the rest of B. It appears that the use of complete, regular and row-regular blocks precludes the identification of reasonable subgroups. When complete blocks are used, there is a tendency to return singletons as one of the subgroups. This suggests weakening such a specification to a regular block. The key problem is that the step away from complete blocks to regular blocks permits a large number of candidate regular blocks of greatly varying densities. This leads to multiple equally well fitting partitions.

If there are subgroups of $\mathrm{A}$ and $\mathrm{B}$, then their attendance patterns must differ if they are to be differentiated. Core women attend more group events than the other women. This suggests blocks of differential densities. To accommodate this notion, we pre-specify the following ideal blockmodel where 'den' indicated that a density is to be specified:

\begin{tabular}{|ccc|}
\hline den & reg & nul \\
rre & reg & nul \\
nul & rre & nul \\
nul & reg & den \\
nul & rre & rre \\
\hline
\end{tabular}

The density has to specified as high and we selected a density of 0.8 for the blocks specified as 'den'. Under this specification, 'den' indicated that there are women who attend heavily a subset of events for their group. The remaining women of a group attend only some of their group's events. For these women we specify a row-regular block. For common events, we use either regular or row-regular blocks. The third row of the blockmodel is specified for Dorothy and Pearl. When this pre-specified blockmodel is fitted with 100,000 repetitions and the heavy penalty (100) for null blocks, we get the unique partition ${ }^{7}$ shown in Table 5.

The partition in Table 5 is quite appealing. Evelyn, Laura and Brenda have heavy participation in events $E_{1}-E_{5}$. While Theresa also has a heavy participation in these events, she does not attend $E_{1}$ and is located in the second cluster of women in Group A. In a similar fashion, it seems that Katherine and Nora form the core of Group B with their heavy attendance of events $E_{10}-E_{14}$, the events for Group B only. However, there is a lingering doubt that Sylvia ought to be located there also.

An alternative strategy for considering subgroups of Groups A and B is to include a finer grained partition of the events. As a part of this alternative, it seems reasonable to focus on null blocks within the events for Group A and within the events for group B. An inspection of the data suggested the following blockmodel:

\footnotetext{
${ }^{7}$ When the last row of the pre-specified blockmodel has null, row-regular, and regular block types specified in that order, the identical unique partition is returned.
} 
Table 5: (5,3)-Partition for Subgroups Using Density Blocks

\begin{tabular}{|l|ccccc|cccc|ccccc|}
\hline Actor & $E_{1}$ & $E_{2}$ & $E_{3}$ & $E_{4}$ & $E_{5}$ & $E_{6}$ & $E_{7}$ & $E_{8}$ & $E_{9}$ & $E_{10}$ & $E_{11}$ & $E_{12}$ & $E_{13}$ & $E_{14}$ \\
\hline Evelyn & 1 & 1 & 1 & 1 & 1 & 1 & 0 & 1 & 1 & 0 & 0 & 0 & 0 & 0 \\
Laura & 1 & 1 & 1 & 0 & 1 & 1 & 1 & 1 & 0 & 0 & 0 & 0 & 0 & 0 \\
Brenda & 1 & 0 & 1 & 1 & 1 & 1 & 1 & 1 & 0 & 0 & 0 & 0 & 0 & 0 \\
\hline Theresa & 0 & 1 & 1 & 1 & 1 & 1 & 1 & 1 & 1 & 0 & 0 & 0 & 0 & 0 \\
Charlotte & 0 & 0 & 1 & 1 & 1 & 0 & 1 & 0 & 0 & 0 & 0 & 0 & 0 & 0 \\
Frances & 0 & 0 & 1 & 0 & 1 & 1 & 0 & 1 & 0 & 0 & 0 & 0 & 0 & 0 \\
Eleanor & 0 & 0 & 0 & 0 & 1 & 1 & 1 & 1 & 0 & 0 & 0 & 0 & 0 & 0 \\
Ruth & 0 & 0 & 0 & 0 & 1 & 0 & 1 & 1 & 1 & 0 & 0 & 0 & 0 & 0 \\
\hline Pearl & 0 & 0 & 0 & 0 & 0 & 1 & 0 & 1 & 1 & 0 & 0 & 0 & 0 & 0 \\
Dorothy & 0 & 0 & 0 & 0 & 0 & 0 & 0 & 1 & 1 & 0 & 0 & 0 & 0 & 0 \\
\hline Sylvia & 0 & 0 & 0 & 0 & 0 & 0 & 1 & 1 & 1 & 1 & 0 & 1 & 1 & 1 \\
Nora & 0 & 0 & 0 & 0 & 0 & 1 & 1 & 0 & 1 & 1 & 1 & 1 & 1 & 1 \\
\hline Verne & 0 & 0 & 0 & 0 & 0 & 0 & 1 & 1 & 1 & 0 & 0 & 1 & 0 & 0 \\
Myrna & 0 & 0 & 0 & 0 & 0 & 0 & 0 & 1 & 1 & 1 & 0 & 1 & 0 & 0 \\
Katherine & 0 & 0 & 0 & 0 & 0 & 0 & 0 & 1 & 1 & 1 & 0 & 1 & 1 & 1 \\
Helen & 0 & 0 & 0 & 0 & 0 & 0 & 1 & 1 & 0 & 1 & 1 & 1 & 0 & 0 \\
Olivia & 0 & 0 & 0 & 0 & 0 & 0 & 0 & 0 & 1 & 0 & 1 & 0 & 0 & 0 \\
Flora & 0 & 0 & 0 & 0 & 0 & 0 & 0 & 0 & 1 & 0 & 1 & 0 & 0 & 0 \\
\hline
\end{tabular}

Table 6: (5,5)-Partition for Subgroups Using More Event Clusters

\begin{tabular}{|l|cccc|c|cccc|ccc|cc|}
\hline Actor & $E_{1}$ & $E_{2}$ & $E_{3}$ & $E_{4}$ & $E_{5}$ & $E_{6}$ & $E_{7}$ & $E_{8}$ & $E_{9}$ & $E_{10}$ & $E_{11}$ & $E_{12}$ & $E_{13}$ & $E_{14}$ \\
\hline Evelyn & 1 & 1 & 1 & 1 & 1 & 1 & 0 & 1 & 1 & 0 & 0 & 0 & 0 & 0 \\
Laura & 1 & 1 & 1 & 0 & 1 & 1 & 1 & 1 & 0 & 0 & 0 & 0 & 0 & 0 \\
Theresa & 0 & 1 & 1 & 1 & 1 & 1 & 1 & 1 & 1 & 0 & 0 & 0 & 0 & 0 \\
Brenda & 1 & 0 & 1 & 1 & 1 & 1 & 1 & 1 & 0 & 0 & 0 & 0 & 0 & 0 \\
Charlotte & 0 & 0 & 1 & 1 & 1 & 0 & 1 & 0 & 0 & 0 & 0 & 0 & 0 & 0 \\
Frances & 0 & 0 & 1 & 0 & 1 & 1 & 0 & 1 & 0 & 0 & 0 & 0 & 0 & 0 \\
\hline Eleanor & 0 & 0 & 0 & 0 & 1 & 1 & 1 & 1 & 0 & 0 & 0 & 0 & 0 & 0 \\
Ruth & 0 & 0 & 0 & 0 & 1 & 0 & 1 & 1 & 1 & 0 & 0 & 0 & 0 & 0 \\
\hline Pearl & 0 & 0 & 0 & 0 & 0 & 1 & 0 & 1 & 1 & 0 & 0 & 0 & 0 & 0 \\
Dorothy & 0 & 0 & 0 & 0 & 0 & 0 & 0 & 1 & 1 & 0 & 0 & 0 & 0 & 0 \\
\hline Katherine & 0 & 0 & 0 & 0 & 0 & 0 & 0 & 1 & 1 & 1 & 0 & 1 & 1 & 1 \\
Sylvia & 0 & 0 & 0 & 0 & 0 & 0 & 1 & 1 & 1 & 1 & 0 & 1 & 1 & 1 \\
Nora & 0 & 0 & 0 & 0 & 0 & 1 & 1 & 0 & 1 & 1 & 1 & 1 & 1 & 1 \\
\hline Verne & 0 & 0 & 0 & 0 & 0 & 0 & 1 & 1 & 1 & 0 & 0 & 1 & 0 & 0 \\
Myrna & 0 & 0 & 0 & 0 & 0 & 0 & 0 & 1 & 1 & 1 & 0 & 1 & 0 & 0 \\
Helen & 0 & 0 & 0 & 0 & 0 & 0 & 1 & 1 & 0 & 1 & 1 & 1 & 0 & 0 \\
Olivia & 0 & 0 & 0 & 0 & 0 & 0 & 0 & 0 & 1 & 0 & 1 & 0 & 0 & 0 \\
Flora & 0 & 0 & 0 & 0 & 0 & 0 & 0 & 0 & 1 & 0 & 1 & 0 & 0 & 0 \\
\hline
\end{tabular}




\begin{tabular}{|ccccc|}
\hline reg & com & reg & nul & nul \\
nul & com & reg & nul & nul \\
nul & nul & rre & nul & nul \\
nul & nul & reg & reg & com \\
nul & nul & rre & reg & nul \\
\hline
\end{tabular}

When this blockmodel is fitted over 50,000 repetitions, the blockmodel shown in Table 6 results. The partition is exact with zero inconsistencies. Event $E_{5}$ is a singleton and all of the women of Group A attend it. For events $E_{1}-E_{4}$, Eleanor and Frances are detached from the rest of Group A by attending none of these events while the remaining members of Group A attend at least one of them. For Group B, Katherine, Sylvia and Nora all attend $E_{13}$ and $E_{14}$ while the members of this group do not attend them.

Each of the two partitions (in Table 5 and Table 6) delineates slightly different features of the internal structure of the two larger groups of women in the Deep South data. While other partitions might be possible, it may well be the case, that this is about all that can be extracted without resorting to extremely fine grained partitions. The additional density block type was useful in establishing a unique exact fitting partition without expanding the number of event clusters.

\section{Thinking of One-Mode Data as if They were Two-Mode Data}

Recognizing that the row and column partitions may be different makes it possible to view one-mode network data in a different fashion by thinking of them as two-mode data. People in an organization can seek advice from each other and form advice networks. Because seeking advice and providing advice are directional activities, the advice networks that they generate will be asymmetric ${ }^{8}$. This suggests that partitioning the rows and columns in different ways will be useful. For social service agencies that refer clients, the act of referral is asymmetric. As a result, these inter-agency one-mode networks can be examined fruitfully as if they were two-mode networks. A final example comes in the form of journalto-journal networks for academic disciplines. Blockmodeling has been used to partition such networks as one-mode networks in terms of structural equivalence. While the partitions had value, there is a sense in which a partitioning strategy for one-mode data is overly restrictive. If there are 'consumer' journals (as the first set of actors) and 'producer' journals (as the second set of actors), it makes sense to think of partitioning the rows and columns of such citation networks in different ways.

The following discussion requires the use of some of the expanded set of block types presented by Doreian et al. $(1994,2004)$. Table 7 shows these block types and, from them, we concentrate on expanding the set of null, complete, and regular blocks with the row-regular, column-regular and rowfunction blocks.

\subsection{Journal-to-Journal Citation Networks}

Journal networks are defined with journals as the vertices and have valued elements. These elements are counts of the number of times articles on one journal cite articles in another journal. The diagonal elements of these (one-mode) journal networks are counts of self-citation for the journals in the network. These (valued) networks have been studied as one-mode networks and partitioned in terms of structural equivalence (Doreian, 1985, 1988). In these partitioning efforts, the rows and columns are clustered in the same way and the diagonal elements are ignored. Such networks have also been studied as one-mode core-periphery structures (Borgatti and Everett, 1999). At face value, these one-mode networks are better viewed as two-mode networks. When this is done, journals are seen as both 'consuming journals' (in the rows as citing journals) and 'producing journals' (in the columns as cited journals). And, consistent with

\footnotetext{
${ }^{8}$ If some pairs of actors seek and provide advice to each other, then the advice network will be non-symmetric.
} 
Table 7: Examples of Blocks with Types of Connections

\begin{tabular}{|c|c|c|c|c|c|c|c|c|c|c|c|c|c|c|c|c|c|}
\hline & \multicolumn{5}{|c|}{$Y$} & & \multicolumn{5}{|c|}{$Y$} & & \multicolumn{5}{|c|}{$Y$} \\
\hline & 1 & & 1 & 1 & & & 0 & 1 & 0 & 0 & & & 0 & 0 & & 0 & \\
\hline$X$ & 1 & 1 & 1 & 1 & 1 & $X$ & 1 & 1 & 1 & 1 & 1 & $X$ & 0 & 0 & 1 & 1 & 0 \\
\hline & 1 & 1 & 1 & 1 & 1 & & 0 & 0 & 0 & 0 & 0 & & & 1 & 1 & 0 & 0 \\
\hline & 1 & 1 & 1 & 1 & 1 & & 0 & 0 & 0 & 1 & 0 & & 0 & 0 & 1 & 0 & 1 \\
\hline
\end{tabular}

\begin{tabular}{|c|c|c|c|c|c|c|c|c|c|c|c|c|c|c|c|c|c|}
\hline & \multicolumn{5}{|c|}{$Y$} & & \multicolumn{5}{|c|}{$Y$} & & \multicolumn{5}{|c|}{$Y$} \\
\hline & 0 & & 0 & 0 & & & 0 & & 0 & 0 & 0 & & $\overline{0}$ & 1 & $\overline{0}$ & 1 & \\
\hline$X$ & 1 & 0 & 1 & 1 & 0 & $X$ & 0 & 1 & 1 & 0 & 0 & $X$ & 1 & 0 & 1 & 0 & 0 \\
\hline & 0 & 0 & 1 & 0 & 1 & & 1 & 0 & 1 & 0 & 0 & & 1 & 1 & 0 & 1 & 1 \\
\hline & 1 & 1 & 0 & 0 & 0 & & 0 & 1 & 0 & 0 & 1 & & 0 & 0 & 0 & 0 & 0 \\
\hline
\end{tabular}

regular

row-regular

col-regular

\begin{tabular}{l|lllll|} 
& \multicolumn{5}{|c|}{$Y$} \\
\hline$X$ & 0 & 0 & 0 & 0 & 0 \\
& 0 & 0 & 0 & 0 & 0 \\
& 0 & 0 & 0 & 0 & 0 \\
& 0 & 0 & 0 & 0 & 0 \\
\hline
\end{tabular}

null

\begin{tabular}{l|lllll|} 
& \multicolumn{5}{|c|}{$Y$} \\
\hline$X$ & 0 & 0 & 0 & 1 & 0 \\
& 0 & 0 & 1 & 0 & 0 \\
& 1 & 0 & 0 & 0 & 0 \\
& 0 & 0 & 0 & 1 & 0 \\
\hline
\end{tabular}

row-functional

\begin{tabular}{c|cccc|} 
& & $Y$ & & \\
\hline \multirow{4}{*}{$X$} & 1 & 0 & 0 & 0 \\
& 0 & 1 & 0 & 0 \\
& 0 & 0 & 1 & 0 \\
& 0 & 0 & 0 & 0 \\
& 0 & 0 & 0 & 1 \\
\hline
\end{tabular}

col-functional

the approach taken in this paper, the rows and columns can be clustered in different ways. Additionally, the self-citation entries (diagonal elements) can be included as an integral part of the data even though the 'diagonal' elements of the image have no meaning.

Viewing citation networks in this fashion allows us to examine the different roles that journals can perform as producers and consumers of knowledge. This can be approached as a simple form of 'hypothesis testing'. If there is no difference between these roles, then the rows and columns, as an empirical matter, will be clustered in the same way. To the extent that rows and columns are clustered differently, we have evidence that journals function differently with regard to the production and consumption of disciplinary knowledge.

In approaching journal networks in this way, we had only some intuitions at the outset. We thought that there would be a core consumer position and a core producer position, consistent with the idea of distinguishing cores from non-cores. Further, to reflect 'coreness', the block corresponding to the two (producer and consumer) cores should be complete. Every core-consumer journal ought to cite every core-producer journal and every core producer journal ought to be cited by every core consumer journal. Second, there will be non-core consumers and non-core producers. Some will vary in the extent to which they approximate a core and others will not be core journals. We speculate that a second level of consumer journals will occupy a position where the block defined by them and the core-producer journals is weaker than a complete block. We specify that the $(1,2)$ block is regular. Put differently, for such a set of near-core consumer journals, each will cite at least one core producer journal and every core producer journal will be cited by at least one the second level of near-core consumer journals. In a similar fashion, 
Table 8: Journals in Social Work Citation Network

\begin{tabular}{|l|r|c|}
\hline Title & Label & Id \\
\hline Administration in Social Work & ASW & m \\
British Journal of Social Work & BJSW & n \\
Child Abuse and Neglect & CAN & j \\
Chile Care Quarterly & CCQ & o \\
Child Welfare & CW & a \\
Children and Youth Services Review & CYSR & b \\
Clinical Social Work Journal & CSWJ & k \\
Family Relations & FR & 1 \\
Journal of Gerontological Social Work & JGSW & p \\
Journal of Social Policy & JSP & q \\
Journal of Social Work Education & JSWE & e \\
Public Welfare & PW & r \\
Social Casework & SCW & c \\
Social Services Review & SSR & f \\
Social Work & SW & d \\
Social Work with Groups & SWG & g \\
Social Work in Health Care & SWHC & h \\
Social Work Research and Abstracts & SWRA & i \\
\hline
\end{tabular}

we think there will be a second level of producer journals whose block with the core consumer journals would be regular -i.e. the $(2,1)$ block is a regular block. Third, weaker consumer and producer positions will have blocks, involving the core producer and core consumer journals, that would be row regular and column regular. Fourth, there will be null blocks. It seems reasonable to expect 'holes' in the structure if journals occupy different niches. There will be sets of consumer journals that never cite some of the producer journals and there will sets of producer journals that are not cited by sets of consumer journals. Finally, we need to allow for certain 'almost null' blocks: some 'diagonal' elements might cause some problems by appearing in erstwhile null blocks. If there are consumer journals that cite some of the core journals and then only themselves, these self-citation counts will appear in null blocks. This suggested the use of the row-function block type to allow a single non-zero element to appear in each row of such a null block. The specified model is:

\begin{tabular}{|cccc|}
\hline com & reg & cre & rre \\
reg & reg & nul & nul \\
reg & nul & rfn & nul \\
rre & rre & nul & rfn \\
cre & nul & nul & rfn \\
\hline
\end{tabular}

With the above arguments in mind, we re-analyze the social work journal network reported by Baker (1992) and analyzed by Borgatti and Everett (1999). The included journals are listed in Table 5. In addition to thinking in terms of blockmodeling these data as two-mode data, there one important difference between out approach and that of Borgatti and Everett. The original data are not symmetric and, in contrast to Borgatti and Everett, we do not symmetrize them for a simple reason. When the data are symmetrized there can be no distinction between production of, and consumption of, knowledge. A twomode analysis of such a symmetrized citation network would yield the same partition of the rows and 
Table 9: Journal Citation Matrix with the (5,4)-Partition

\begin{tabular}{|l|c|rrrr|rrrrr|rrr|rrrrrr|}
\hline & $\mathrm{Id}$ & $\mathrm{a}$ & $\mathrm{c}$ & $\mathrm{f}$ & $\mathrm{d}$ & $\mathrm{m}$ & $\mathrm{e}$ & $\mathrm{g}$ & $\mathrm{h}$ & $\mathrm{i}$ & $\mathrm{j}$ & $\mathrm{k}$ & $\mathrm{l}$ & $\mathrm{n}$ & $\mathrm{o}$ & $\mathrm{b}$ & $\mathrm{p}$ & $\mathrm{q}$ & $\mathrm{r}$ \\
\hline CW & $\mathrm{a}$ & 187 & 32 & 10 & 58 & 0 & 11 & 0 & 0 & 0 & 7 & 0 & 0 & 0 & 0 & 6 & 0 & 0 & 7 \\
CYSR & $\mathrm{b}$ & 70 & 8 & 14 & 28 & 0 & 0 & 0 & 0 & 5 & 12 & 0 & 0 & 0 & 5 & 26 & 0 & 0 & 6 \\
SCW & $\mathrm{c}$ & 17 & 149 & 36 & 124 & 8 & 21 & 8 & 6 & 18 & 6 & 8 & 6 & 0 & 0 & 0 & 6 & 0 & 0 \\
SW & $\mathrm{d}$ & 52 & 58 & 53 & 356 & 15 & 33 & 15 & 43 & 8 & 0 & 0 & 9 & 0 & 0 & 0 & 0 & 0 & 19 \\
\hline JSWE & $\mathrm{e}$ & 0 & 18 & 16 & 58 & 9 & 104 & 0 & 7 & 16 & 0 & 0 & 0 & 0 & 0 & 0 & 0 & 0 & 0 \\
SSR & $\mathrm{f}$ & 17 & 30 & 105 & 106 & 7 & 9 & 0 & 0 & 25 & 0 & 0 & 0 & 0 & 0 & 0 & 0 & 0 & 0 \\
SWG & $\mathrm{g}$ & 0 & 9 & 7 & 40 & 0 & 9 & 41 & 9 & 0 & 0 & 0 & 0 & 0 & 0 & 0 & 0 & 0 & 0 \\
SWHC & $\mathrm{h}$ & 0 & 20 & 0 & 26 & 0 & 0 & 0 & 86 & 0 & 0 & 0 & 0 & 0 & 0 & 0 & 0 & 0 & 0 \\
SWRA & $\mathrm{i}$ & 8 & 8 & 39 & 44 & 0 & 24 & 0 & 0 & 40 & 0 & 0 & 0 & 0 & 0 & 0 & 0 & 0 & 0 \\
\hline CAN & $\mathrm{j}$ & 9 & 6 & 0 & 8 & 0 & 0 & 0 & 0 & 0 & 109 & 0 & 0 & 0 & 0 & 0 & 0 & 0 & 0 \\
CSWJ & $\mathrm{k}$ & 0 & 47 & 20 & 45 & 0 & 0 & 0 & 0 & 0 & 0 & 40 & 0 & 0 & 0 & 0 & 0 & 0 & 0 \\
FR & $\mathrm{l}$ & 0 & 18 & 0 & 9 & 0 & 0 & 0 & 0 & 0 & 0 & 0 & 205 & 0 & 0 & 0 & 0 & 0 & 0 \\
\hline ASW & $\mathrm{m}$ & 0 & 0 & 21 & 73 & 70 & 18 & 0 & 0 & 7 & 0 & 0 & 0 & 0 & 0 & 0 & 0 & 0 & 13 \\
BJSW & $\mathrm{n}$ & 0 & 0 & 0 & 19 & 0 & 13 & 0 & 0 & 0 & 0 & 0 & 0 & 95 & 0 & 0 & 0 & 0 & 0 \\
\hline CCQ & $\mathrm{o}$ & 12 & 0 & 0 & 0 & 0 & 0 & 0 & 0 & 0 & 0 & 0 & 0 & 0 & 92 & 0 & 0 & 0 & 0 \\
JGSW & $\mathrm{p}$ & 0 & 16 & 0 & 18 & 0 & 0 & 0 & 0 & 0 & 0 & 0 & 0 & 0 & 0 & 0 & 9 & 0 & 0 \\
JSP & $\mathrm{q}$ & 0 & 0 & 7 & 0 & 0 & 0 & 0 & 0 & 0 & 0 & 0 & 0 & 0 & 0 & 0 & 0 & 35 & 0 \\
PW & $\mathrm{r}$ & 0 & 0 & 0 & 0 & 0 & 0 & 0 & 0 & 0 & 7 & 0 & 0 & 0 & 0 & 0 & 0 & 0 & 9 \\
\hline
\end{tabular}

columns. This is especially true when a symmetrized element is constructed from a non-zero and zero citation rate for a pair of journals.

We leave out two of the journals included by Baker. One is the Indian Journal of Social Work (IJS) really does not belong to the network ${ }^{9}$. It is linked weakly with only one other journal. Also eliminated was Administration in Mental Health because it was linked weakly to only one other journal. This leaves the 18 journals whose citation volumes are shown in Table 9.

Table 9 has been organized so that rows columns that have been permuted to reflect a partitioned structure with five consumer positions and four producer positions. The producer positions (where $P J_{i}$ is used to label producer clusters) are:

$$
\begin{array}{|l|l|}
\hline P J_{1} & \{\mathrm{CW}, \mathrm{SCW}, \mathrm{SSR}, \mathrm{SW}\} \\
P J_{2} & \text { AASW, JSWE, SWG, SWHC, SWRA }\} \\
P J_{3} & \text { \{CAN, CSWJ, FR } \\
P J_{4} & \text { (BJSW, CCQ, CYSR, JGSW, JSP, PW }\} \\
\hline
\end{array}
$$

and the consumer positions (where $C J_{j}$ is used to label the consumer positions) are:

$$
\begin{array}{|l|l|}
\hline C J_{1} & \{\mathrm{CW}, \mathrm{CYSR}, \mathrm{SCW}, \mathrm{SW}\} \\
C J_{2} & \{\text { JSWE, SSR, SWG, SWHC, SWRA }\} \\
C J_{3} & \{\mathrm{CAN}, \mathrm{CSWJ}, \mathrm{FR}\} \\
C J_{4} & \{\text { ASW, BJSW }\} \\
C J_{5} & \{\mathrm{CCQ}, \mathrm{JGSW}, \mathrm{JSP}, \mathrm{PW}\} \\
\hline
\end{array}
$$

It is clear that the consuming positions differ from the producing positions. There is a single inconsistency between the fitted blockmodel and the corresponding ideal blockmodel. This is the bolded element (7) in the in the block defined by the fifth row position and the third column position.

Three journals $(\mathrm{CW}, \mathrm{SCW}, \mathrm{SW})$ belong to both of the core positions - they are core producers in $P J_{1}$ and are core consumers in $C J_{1}$. Social Service Review (SSR) is in $P J_{1}$ but not $C J_{1}$ while Children and Youth Services Review (CYSR) is $C J_{1}$ but is not $C P_{1}$. SSR does appear in the second consumer

\footnotetext{
${ }^{9}$ Consulting members of a social work faculty about these data, all indicated that IJS, regardless of its merits, is not visible in the U.S. and is seldom or never consulted.
} 


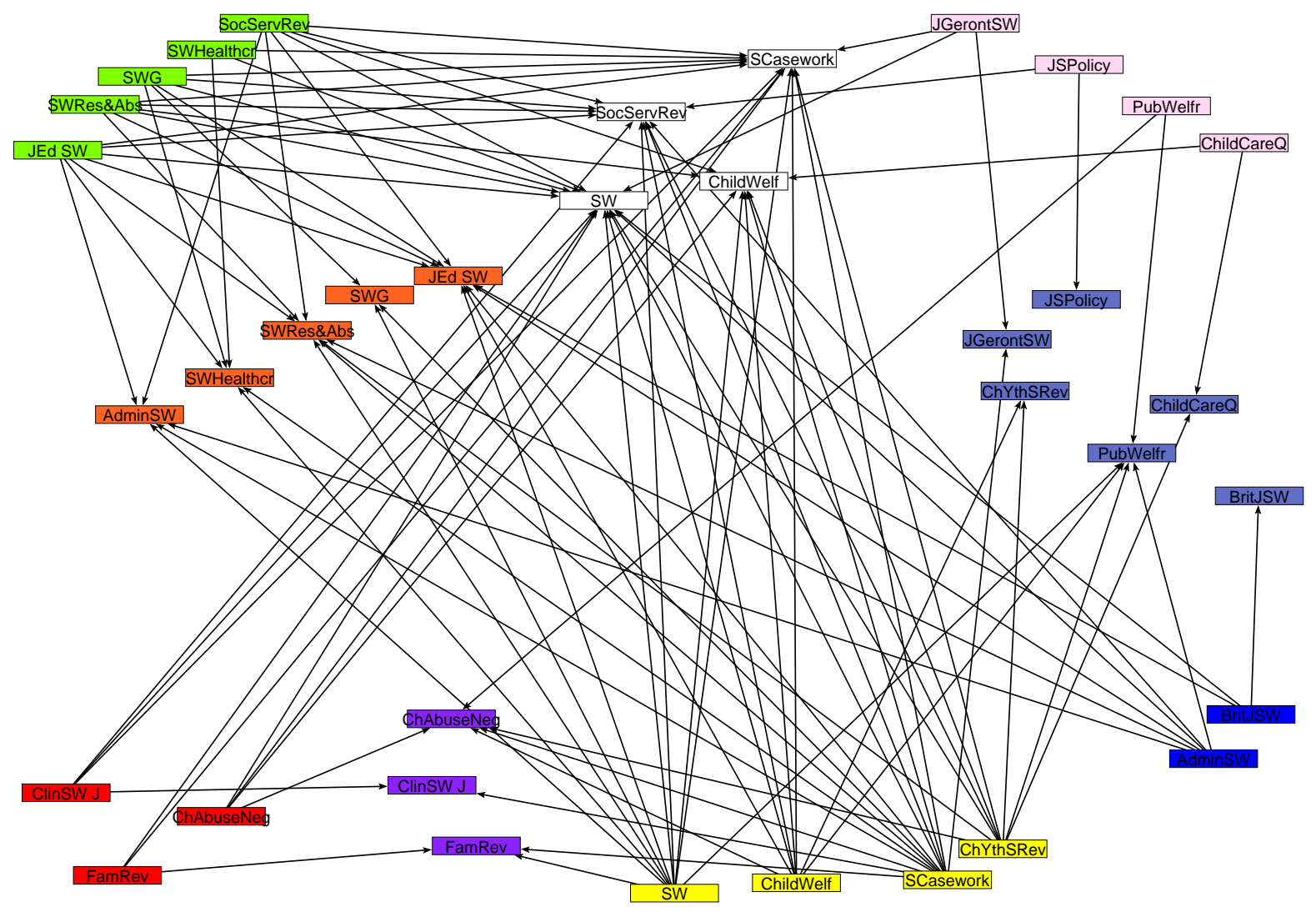

Figure 5: Social Work Journal Bipartite (5,4) Network

position. Four journals, (JSWE, SWG, SWHC, SWRA) are common to the second producer position $\left(P J_{2}\right)$ and the second consumer position $\left(C J_{2}\right)$. Together, these most prominent journals vary in their roles as producers and consumers of social work knowledge. We note that the block defined by $C J_{2}$ and $P J_{2}$ is regular. This is also the case for the block defined by $C J_{1}$ and $P J_{2}$. The third producer position $\left(P J_{3}\right)$ and the third consumer position $\left(C J_{3}\right)$ share the same set of journals, namely, CAN, CSWJ, and FR. The block defined by $C J_{3}$ and $P C_{1}$ regular as is the block defined by $C J_{1}$ and $P C_{3}$ (although we only specified it as row-regular). The block defined by $C J_{3}$ and $P J_{3}$ is a row-functional block. Apart from these block types, the blocks associated with these positions are null blocks. The block defined by $C P_{4}$ and $C J_{1}$ is row-regular while the block defined by $C P_{1}$ and $C J_{5}$ is column-regular. Apart from these, the blocks for the last consumer and producer positions are null or row-functional. The one inconsistency for the fitted blockmodel is the tie from PW to CAN. The bipartite graph (for incidence of ties but not their values) is shown in Figure 5 where the journals in each of the producer and consumer positions are grouped together.

There is an interesting contrast with the analysis of Borgatti and Everett (1999) who found a single core in a core-periphery structure. The core they established was $\{\mathrm{SSR}, \mathrm{SCW}, \mathrm{SW}\}$. These three journals are in our producer core $\left(P J_{1}\right)$ but only SCW and $\mathrm{SW}$ are in the consumer core $\left(C J_{1}\right)$. In our analysis, the producer core includes also CW while the core consumer includes CW and CYSR. Analyzing these data with a two-mode blockmodeling approach provides a more differentiated view of the core. 


\section{Summary and Discussion}

While (generalized) blockmodeling has been used primarily as a powerful way of modeling one-mode network data, we have extended (generalized) blockmodeling to examine two-mode data. To do this, it is necessary to think in terms of partitioning the rows and columns separately as a part of the overall partitioning procedure. Two examples, the Supreme Court data and the Southern Women data, were two-mode data sets. For the former we used structural equivalence in an unrestricted fashion and, for the latter, we used the same equivalence idea but added to it the use of a pre-specified blockmodel.

The partitions returned for both of these data sets are straightforward and compelling. To the extent that the 'true' partition stemming from Freeman's meta-analysis of the many prior analyses of the Southern Women data provides a benchmark, the two-mode blockmodeling approach discussed here does delineate the true structure of the subgroup formation for these data, consistent with the analysis of Freeman and White (1994). Additionally, the corresponding partition of the events is obtained. In the same fashion, the joint partition of justices and decisions of the Supreme Court data provides considerable insight into the political stance of the justices and the events (decisions) that reveal their differences.

The idea of partitioning the rows and columns in different ways is imposed by the nature of two-mode data. There is no other way to proceed. However, the idea of partitioning rows and columns in distinct ways need not be restricted to two-mode data and can be used for one-mode data. For symmetric onemode data structures, there is no reason to use two-mode data analytic methods. However, for asymmetric and non-symmetric one-mode networks, there is much to be gained by viewing them through the twomode lens. The third example, in the form of a journal-to-journal citation network, shows the utility of treating one-mode data as though they were two-mode data. Journals differ in terms of their producer and consumer positions and roles in the communication of knowledge.

\section{References}

[1] Atkin, R. H. (1974). Mathematical Structure in Human Affairs. London: Heinemann Educational Books.

[2] Baker, D. R. (1992). A structural analysis of the social work journal network: 1985-1986. Journal of Social Service Research, 15, 153-167.

[3] Batagelj, V. (1997). Notes on blockmodeling. Social Networks, 19, 143-155.

[4] Batagelj, V., Doreian, P. \& Ferligoj, A. (1992a). An optimizational approach to regular equivalence. Social Networks, 14, 121-135.

[5] Batagelj, V., Ferligoj, A. \& Doreian, P. (1992b). Direct and indirect methods for structural equivalence. Social Networks, 14, 63-90.

[6] Borgatti, S. P. \& Everett, M. G. (1997). Network analysis of 2-mode data. Social Networks, 19, 243-269.

[7] Borgatti, S. P. \& Everett, M. G. (1999). Models of core/priphery structures. Social Networks, 1999, 375-395.

[8] Breiger, R. L. (1974). The duality of persons and groups. Social Forces, 53, 181-190.

[9] Breiger, R. L., Boorman, S. A. \& Arabie, P. (1975). An algorithm for clustering relational data with applications to social network analysis and comparison to multidimensional scaling. Journal of Mathematical Psychology, 12, 328-383. 
[10] Burt, R. S. (1976). Positions in networks. Social Forces, 55, 93-122.

[11] Davis, A., Gardner, B. \& Gardner, M. R. (1941). Deep South. Chicago: University of Chicago Press.

[12] Doreian, P. (1979). On delineation of small group structure. In Hudson, H. C. (Ed.), Classifying Social Data. San Francisco: Jossey-Bass.

[13] Doreian, P. (1985). Structural equivalence in a psychology journal network. Journal of the American Society for Information Science, 36, 411-417.

[14] Doreian, P. (1988). Testing structural equivalence in a network of geographical journals. Journal of the American Society for Information Science, 39, 79-85.

[15] Doreian, P., Batagelj, V. \& Ferligoj, A. (1994). Partitioning networks based on generalized concepts of equivalence. Journal of Mathematical Sociology, 19, 1-27.

[16] Doreian, P. \& Fujimoto, K. (2003). Structures of supreme court voting. Connections, 25(3) (forthcoming).

[17] Everett, M. G. \& Borgatti, S. P. (1993). An extension of regular colouring of graphs to digraphs, networks and hypergraphs. Social Networks, 15, 237-254.

[18] Freeman, L. C. (1980). Q-analysis and the structure of friendship networks. International Journal of Man-Machine Studies, 12, 367-378.

[19] Freeman, L. C. (2002). Finding social groups: A meta-analysis of the southern women data. In Breiger, R., Carley, C., and Pattison, P.Dynamic Social Network Modeling and Analysis: Workshop Summary and Papers (pp 39-97), Washington D.C.: National Research Council, The National Academies Press.

[20] Freeman, L. C. \& White, D. R. (1994). Using Galois lattices to present network data. In Marsden, P. (Ed.), Sociological Methodology (pp. 127-146). Cambridge: Blackwell.

[21] Greenhouse, L. (2001). In year of Florida vote, supreme court did much other work. New York Times, Monday, July 2.

[22] Homans, G. C. (1950). The Human Group. New York: Harcourt, Brace and World.

[23] Lorrain, F. \& White, H. C. (1971). Structural equivalence of individuals in social networks. Journal of Mathematical Sociology, 1, 49-80.

[24] Seidman, S. (1981). Structures induced by collection of subsets. Mathematical Social Sciences, 1, 381-396.

[25] Wasserman, S. \& Faust, K. (1994). Social Network Analysis: Methods and Applications. Cambridge: Cambridge University Press.

[26] White, D. R. \& Reitz, K. P. (1983). Graph and semigroup homomorphisms on networks of relations. Social Networks, 5, 193-234. 